\title{
What do babies eat? Evaluation of a food frequency questionnaire to assess the diets of infants aged 6 months
}

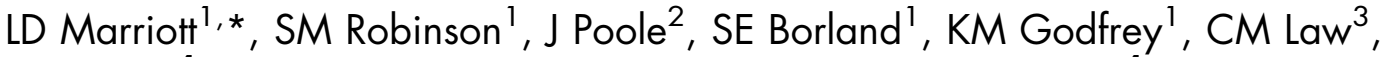 \\ HM Inskip ${ }^{1}$ and The Southampton Women's Survey Study Group' \\ ${ }^{1}$ MRC Epidemiology Resource Centre, University of Southampton, Southampton SO16 6YD, UK: ${ }^{2}$ Trent Cancer \\ Registry, Sheffield S10 3TG, UK: ${ }^{3}$ Centre for Paediatric Epidemiology and Biostatistics, Institute of Child Health, \\ London WCIN IEH, UK
}

Submitted 30 April 2007: Accepted 9 September 2007: First published online 16 November 2007

\begin{abstract}
Objective: To evaluate the relative validity of a food frequency questionnaire (FFQ) for assessing nutrient intakes in 6-month-old infants.

Design and setting: The FFQ was developed to assess the diets of infants born to women in the Southampton Women's Survey (SWS), a population-based survey of young women and their offspring. The energy and nutrient intakes obtained from an interviewer-administered FFQ were compared with those obtained from a 4-day weighed diary.

Subjects and methods: A sub-sample of 50 infants aged 6 months from the SWS had their diets assessed by both methods. The FFQ recorded the frequencies and amounts of milks, baby foods, regular foods and drinks consumed by the infants over the previous seven days. The diaries recorded the weights of all foods and drinks consumed by the infants on four separate days within 15 days following FFQ completion.

Results: Spearman rank correlation coefficients for intakes of energy, macronutrients and 18 micronutrients, determined by the two methods, ranged from $r=0.39$ to 0.86 ; adjustment for energy intake tended to increase the correlation coefficients, range $r_{\mathrm{a}}=0.55$ to 0.89 . Bland-Altman statistics showed that mean differences between methods were in the range of $-12.5 \%$ to $+12 \cdot 5 \%$ except for vitamin $\mathrm{B}_{12}(-18.9 \%)$.

Conclusion: Although there were differences in absolute energy and nutrient intakes between methods, Spearman rank correlation coefficients indicated reasonable agreement in the ranking of intakes. The interviewer-administered FFQ is a useful tool for assessing energy and nutrient intakes of healthy infants aged about 6 months.
\end{abstract}

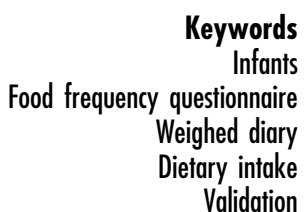

Evidence for the long-term effects of infant nutrition on later health ${ }^{(1)}$ has given impetus to the need to develop methods for assessing the diets of populations of infants. Previous studies in Europe and the USA have mainly used estimated or weighed records to assess diet at around 6 months ${ }^{(2-8)}$. While such methods have been shown to produce ranges of energy intakes that are comparable with levels determined by doubly labelled water (DLW) studies $^{(2)}$, they are expensive to process, often restricted to small groups of subjects and are unlikely to include all sectors of a population, with the consequent possibility of bias. Food frequency questionnaires (FFQs) have been used in older age groups to assess diet in large groups but few researchers have adapted this approach for infants. Two recently published studies using semi-quantitative FFQs, from Norway ${ }^{(9)}$ and Finland ${ }^{(10)}$, have reported food and milk patterns. We are unaware of any studies that report on the validity of energy and nutrient intakes from total milk and food intakes assessed by FFQ in 6-monthold infants.

In a sample of infants aged 6 months we aimed to compare the energy and nutrient intakes assessed by a newly developed, interviewer-administered, infant FFQ with intakes determined from a 4-day weighed diary (WD).

\section{Subjects and methods}

\section{Subjects}

The study was set within the Southampton Women's Survey (SWS) ${ }^{(11)}$, in which women were recruited and 
characterised when not pregnant, then followed through their subsequent pregnancy and the offspring followed-up. The SWS study population of infants born between 1998 and 2003 comprised 1973 infants. Of these, 1645 (83\%) were visited within two weeks of their 6-month birthday. After stratification by current breast-feeding status and infant sex, SWS families were randomly selected to participate in a weighed intake study between September 2001 and March 2003. The dietary intakes of 50 infants assessed both by FFQ and WD are described below. Infants were weighed at both FFQ and diary collection.

\section{The FFQ}

The FFQ was developed to assess the diets of infants aged 6 months $^{(12)}$. It includes meat, fish, vegetables, fruits, cereals and snack foods sub-divided into 16 food groups; 12 categories of commercial baby foods, sub-divided by brand; and six types of non-milk drinks. Human milk, baby formulas and other milk intakes, and age of introduction of solids, were recorded in separate sections.

The questionnaire was completed by a nurse using information supplied by the infant's main carer, during a structured interview lasting about 30 minutes. The daily non-human milk intake in the previous week was estimated from the average total volume of bottle-feeds consumed per day; for human milk, the usual feed length and number of feeds per day were recorded. The frequency of consumption and the amount of each food in the 34-item FFQ consumed over the last week were recorded. Prompt cards were used to show the foods included in each FFQ group to ensure consistent responses; portion size was described using household measures and with the aid of food models. Foods not listed in the FFQ, but consumed in the previous week, were recorded in an open section.

\section{The WD}

A 4-day WD was used as the method of establishing the relative validity of the FFQ. Calculations of inter-individual and intra-individual variation from energy intake data $^{(13)}$, for infants aged 6 months, demonstrated that four days of recording is sufficient to classify more than $75 \%$ of infants into the correct quintile of intake at this age; and is consistent with the diary lengths chosen for recent dietary intake studies in young children ${ }^{(2,14-16)}$. Each family was provided with a diary and Soehnle scales accurate to $1 \mathrm{~g}$. At a home visit, the infant's main carer was instructed in diary completion. Carers were contacted during diary completion to address any problems and the WD was checked at collection to resolve queries. All nonhuman milks, foods and drinks consumed by the infant on each of any four days in the next week were recorded by weight and cooking method. For breast-fed infants, the length of each feed was recorded. In a few instances, the carer estimated the amount of some foods. Additionally, the carer rated each day's intake on how 'typical' it was.

\section{Dietary analysis}

For the FFQ and WD, human milk intake was estimated using an algorithm based on length of suckling derived from published intake data ${ }^{(6,17)}$. Nutritional composition information for infant formulas and commercial baby foods was obtained from manufacturers and/or by calculation from ingredients; and for non-baby products was taken from McCance \& Widdowson's The Composition of Foods, 5th edition and supplements ${ }^{(18)}$. Nutrient data for all foods, milks and other drinks in the FFQ and WD were completed for energy, macronutrients and 18 micronutrients. Nutrient intakes from dietary supplements were excluded.

\section{Statistical methods}

Spearman's rank correlation coefficients were used to summarise the association between the two assessments of each dietary variable. All nutrients were then adjusted for energy intake using the method of Willett ${ }^{(19)}$ and the correlation coefficients recalculated. Bland-Altman plots ${ }^{(20)}$ were produced to assess the levels of agreement between the methods. As the distributions of all nutrients were skewed, logarithmic transformations were used prior to the Bland-Altman analysis and the results are presented on a $\log$ scale. The Bland-Altman limits of agreement are expressed as symmetric percentages, which summarise the percentage differences between the two methods ${ }^{(21)}$. Regression analysis was used to explore whether there were specific factors that influenced the difference between energy intakes assessed by FFQ and WD.

\section{Results}

No statistically significant differences were found between the characteristics of the 50 mother-infant pairs involved in diary completion and those in the SWS cohort who provided FFQ information only (Table 1). The median interval between FFQ completion and the beginning of the WD was $7 \mathrm{~d} ; 84 \%$ of diaries were completed on four consecutive days, the remainder were finished within $8 \mathrm{~d}$; one diary detailed consumption for $3 \mathrm{~d}$ only.

\section{Comparison of FFQ with WD}

There was close similarity between intakes measured by FFQ and WD, with reasonable to good correlation coefficients ranging from $r=0.39$ to 0.86 (Table 2). The FFQ typically gave higher median intake values than the WD, except for sodium. The correlation coefficients 
Table 1 Characteristics of mother-infant pairs who completed a 4-day weighed diary (WD) and the remainder of the cohort who completed a food frequency questionnaire (FFQ)

\begin{tabular}{|c|c|c|c|}
\hline Characteristic & FFQ $(n=1595)^{\star}$ & WD $(n=50)$ & $P$ \\
\hline \multicolumn{4}{|l|}{ Maternal } \\
\hline Age at child's birth (years), median (IQR) & $30(27,33)$ & $30(28,33)$ & 0.36 \\
\hline Body mass index $\left(\mathrm{kg} / \mathrm{m}^{2}\right)$, median (IQR) & $24.3(22 \cdot 0,27 \cdot 7)$ & $24.1(22.4,26.0)$ & 0.71 \\
\hline \multicolumn{4}{|l|}{ Education, \% $(n)$} \\
\hline Up to CSE & $12.9(206)$ & $8.0(4)$ & 0.75 \\
\hline O and/or A levels & $57.9(921)$ & $60.0(30)$ & \\
\hline HND or degree & $29.4(464)$ & $32 \cdot 0(16)$ & \\
\hline Smokers, \% (n) & $27 \cdot 2(433)$ & $26 \cdot 0(13)$ & $0 \cdot 86$ \\
\hline \multicolumn{4}{|l|}{ Infant } \\
\hline Birth weight $(\mathrm{kg})$, median (IQR) & $3.46(3.15,3.80)$ & $3.50(3.20,3.76)$ & 0.90 \\
\hline Weight at FFQ (kg), median (IQR) & $7.93(7.30,8.54)$ & $7.96(7.42,8.58)$ & 0.79 \\
\hline Sex ratio (girls), \% $(n)$ & $46 \cdot 0(732)$ & $50 \cdot 0(25)$ & 0.57 \\
\hline Still breast-fed, \% (n) & $29.7(474)$ & $28.0(14)$ & 0.79 \\
\hline Age at solid food introduction (weeks), median (IQR) & $17.4(15.0,18.4)$ & $17.4(16 \cdot 0,18.4)$ & 0.46 \\
\hline \multicolumn{4}{|l|}{ Birth order, \% $(n)$} \\
\hline 1st & $46 \cdot 2(736)$ & $44.0(22)$ & 0.83 \\
\hline $2 \mathrm{nd} / 3 \mathrm{rd}$ & $49.4(788)$ & $50.0(25)$ & \\
\hline 4th or above & $4.4(70)$ & $6.0(3)$ & \\
\hline
\end{tabular}

IQR, interquartile range.

*Total number does not always sum to 1595 due to missing data.

Table 2 Energy and nutrient intakes estimated by the food frequency questionnaire (FFQ) and the 4-day weighed diary (WD), Spearman rank correlation coefficients $(r)$, energy-adjusted correlation coefficients $\left(r_{\mathrm{a}}\right)$, and Bland-Altman statistics

\begin{tabular}{|c|c|c|c|c|c|c|}
\hline \multirow[b]{2}{*}{ Energy/nutrient } & \multicolumn{2}{|l|}{ FFQ } & \multirow[b]{2}{*}{$r^{*}$} & \multirow[b]{2}{*}{$r_{\mathrm{a}}{ }^{\dagger}$} & \multicolumn{2}{|c|}{ Bland-Altman } \\
\hline & Median (IQR) & Median (IQR) & & & $\begin{array}{l}\text { Mean difference, } \\
\text { FFQ minus WD (\%) }\end{array}$ & $\begin{array}{l}\text { Limits of } \\
\text { agreement }(\%)\end{array}$ \\
\hline Energy (kJ) & $3329(2804,3792)$ & $2968(2728,3423)$ & 0.41 & $\mathrm{n} / \mathrm{a}$ & $6 \cdot 2$ & $-40,52$ \\
\hline Energy/kg body weight (kJ) & $424(366,486)$ & $370(342,423)$ & 0.43 & $\mathrm{n} / \mathrm{a}$ & 8.9 & $-37,55$ \\
\hline Protein $(\mathrm{g})$ & $20 \cdot 6(17 \cdot 9,24.3)$ & $19.7(17.4,23.6)$ & 0.51 & 0.71 & 0.6 & $-48,49$ \\
\hline Fat $(\mathrm{g})$ & $31.3(28.4,37.3)$ & $31.3(27.6,34.7)$ & 0.39 & 0.70 & 3.6 & $-47,54$ \\
\hline Carbohydrate (g) & $106.0(86.5,118.5)$ & $93.1(81.1,106.9)$ & 0.51 & 0.66 & $9 \cdot 2$ & $-37,55$ \\
\hline Total sugars $(\mathrm{g})$ & $77.4(65.7,86.3)$ & $69.4(63.0,81 \cdot 1)$ & 0.55 & 0.69 & $9 \cdot 1$ & $-36,54$ \\
\hline Sodium (mg) & $248(199,305)$ & $270(193,354)$ & 0.60 & 0.56 & $-12 \cdot 2$ & $-87,62$ \\
\hline Potassium (mg) & $1035(871,1205)$ & $1014(791,1137)$ & 0.56 & 0.70 & 4.5 & $-39,48$ \\
\hline Calcium (mg) & $547(462,668)$ & $543(419,726)$ & 0.62 & 0.78 & 3.4 & $-58,65$ \\
\hline Magnesium (mg) & $86.7(67.4,98.1)$ & $78.7(63.7,92.7)$ & 0.66 & 0.68 & 7.9 & $-38,54$ \\
\hline Phosphorus (mg) & $422(330,534)$ & $409(318,531)$ & 0.66 & 0.77 & -0.4 & $-56,56$ \\
\hline Iron (mg) & $7.0(4.9,9.1)$ & $6.9(4.9,8.7)$ & 0.75 & 0.78 & $2 \cdot 0$ & $-59,63$ \\
\hline Zinc (mg) & $5 \cdot 2(4.2,5.9)$ & $4.7(3.8,5.5)$ & 0.69 & 0.83 & 7.4 & $-44,59$ \\
\hline Copper (mg) & $0.46(0.40,0.51)$ & $0.41(0.36,0.49)$ & 0.47 & 0.55 & $5 \cdot 1$ & $-42,52$ \\
\hline Retinol $(\mu \mathrm{g})$ & $597(490,749)$ & $531(462,623)$ & 0.44 & 0.76 & $10 \cdot 0$ & $-49,69$ \\
\hline Vitamin D ( $\mu \mathrm{g})$ & $9.3(5.9,12.3)$ & $8.3(3.7,10.0)$ & 0.86 & 0.83 & $9 \cdot 7$ & $-71,90$ \\
\hline Vitamin E (mg) & $6.0(4.6,8.4)$ & $5.3(4.5,6.9)$ & 0.76 & 0.83 & 11.3 & $-44,67$ \\
\hline Thiamin (mg) & $0.65(0.49,0.89)$ & $0.60(0.46,0.86)$ & 0.85 & 0.89 & $9 \cdot 3$ & $-44,63$ \\
\hline Riboflavin (mg) & $1.2(0.6,1.3)$ & $1.2(0.7,1.4)$ & 0.78 & 0.87 & $-2 \cdot 6$ & $-57,52$ \\
\hline Niacin (mg) & $8.1(5.4,11.3)$ & $8.0(5 \cdot 3,9 \cdot 3)$ & 0.86 & 0.88 & $7 \cdot 3$ & $-38,53$ \\
\hline Vitamin $B_{6}(\mathrm{mg})$ & $0.60(0.45,0.79)$ & $0.60(0.48,0.72)$ & 0.81 & 0.84 & 3.2 & $-43,50$ \\
\hline Folic acid $(\mu \mathrm{g})$ & $112(88,122)$ & $100(78,121)$ & 0.68 & 0.65 & $9 \cdot 1$ & $-42,60$ \\
\hline Vitamin $B_{12}(\mu \mathrm{q})$ & $1.8(1.3,2.1)$ & $1.8(1.4,2.2)$ & 0.75 & 0.74 & -18.9 & $-135,97$ \\
\hline Vitamin C (mg) & $86(70,109)$ & $78(68,90)$ & 0.67 & 0.67 & 11.2 & $-45,67$ \\
\hline
\end{tabular}

n/a, not applicable.

${ }^{*}$ All significantly different from zero, $P<0.001$, except energy $(P=0.003)$, fat $(P=0.005)$ and retinol $(P=0.0013)$.

tAll significantly different from zero, $P<0.001$.

‡Limits are expressed as symmetric percentage differences between FFQ and WD values.

were strengthened by adjustment for energy intake, range $r_{\mathrm{a}}=0.55$ to 0.89 . In the exploration of differences in absolute intakes of energy and nutrients between the FFQ and WD by Bland-Altman plots, most mean differences were positive and all within the range of $-12.5 \%$ to $+12.5 \%$ except for vitamin $B_{12},-18.9 \%$ (Table 2 ).

\section{Exploration of differences in intakes}

The between-methods Spearman rank correlation coefficient for energy intake was lower $(r=0.28)$ for the $28 \%$ of infants who were still breast-fed at FFQ completion than for infants who were not receiving human milk $(r=0.48)$, a possible consequence of the imprecision of 


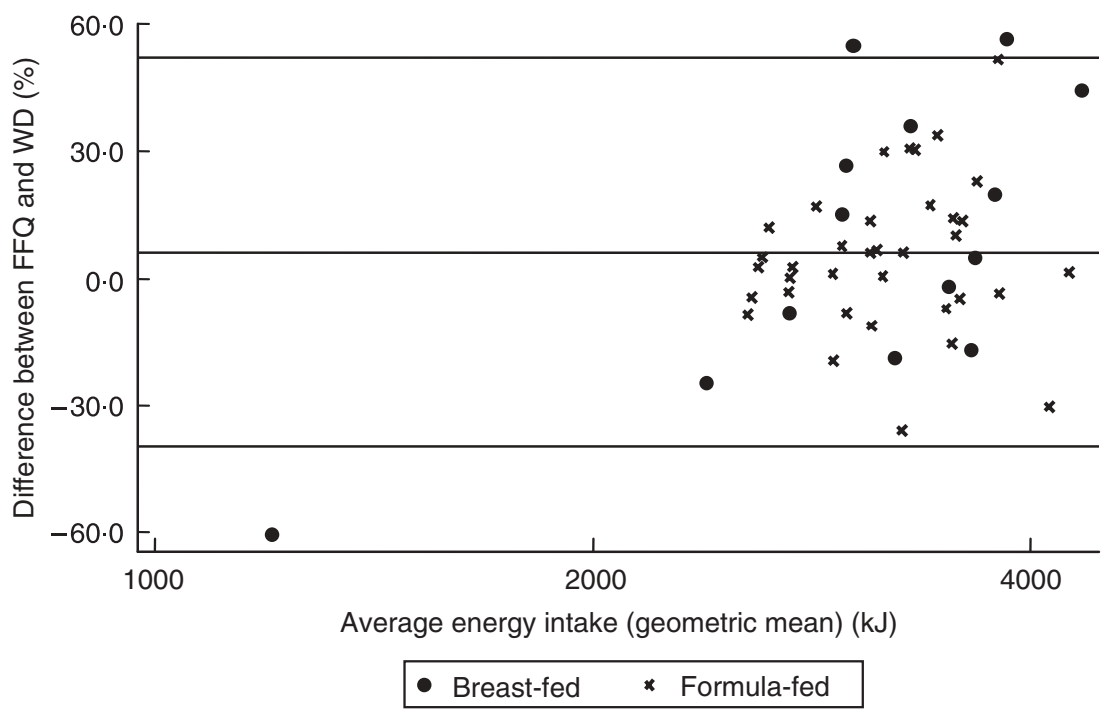

Fig. 1 Bland-Altman plot assessing the agreement for energy intakes obtained with the food frequency questionnaire (FFQ) and the 4-day weighed diary (WD), differentiated by milk feeding. Filled circles represent infants still breast-fed at FFQ completion; crosses represent formula-fed infants

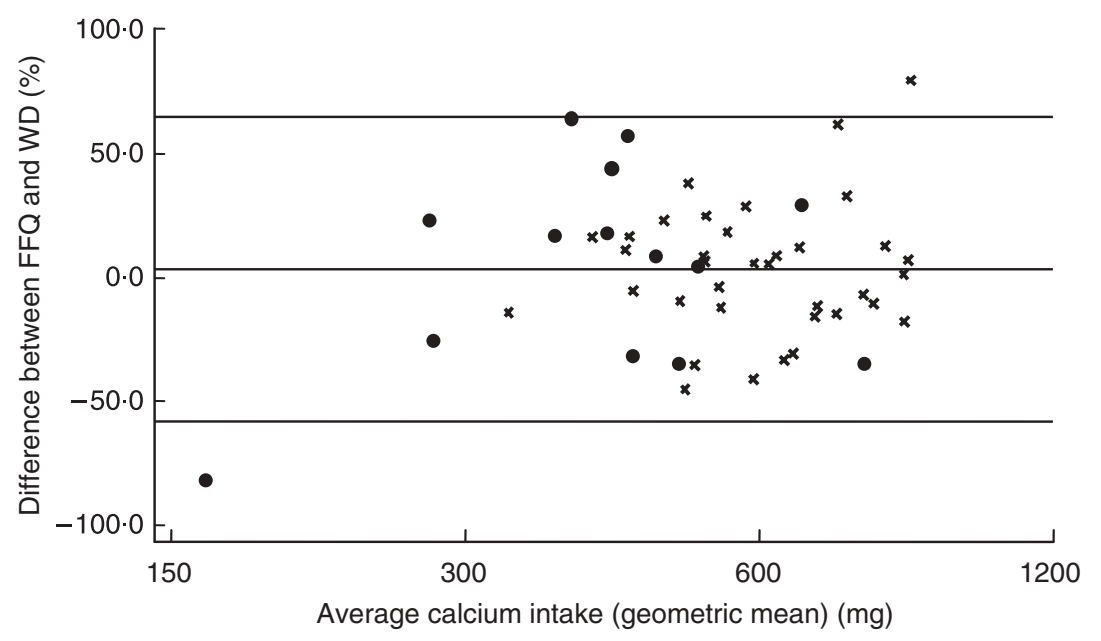

- Breast-fed $\quad \times$ Formula-fed

Fig. 2 Bland-Altman plot assessing the agreement for calcium intakes obtained with the food frequency questionnaire (FFQ) and the 4-day weighed diary (WD), differentiated by milk feeding. Filled circles represent infants still breast-fed at FFQ completion; crosses represent formula-fed infants

human milk intake estimation. Additionally, in the BlandAltman plots (Figs 1 and 2) the distributions of points for the 'breast-fed' infants were more widely spread than those for the 'formula-fed' infants. However, univariate regression analysis of the differences in energy intakes from the two assessments showed no association with whether the infant was still breast-fed at 6 months $(P=0 \cdot 24)$

Univariate regression analysis was used to investigate factors that might influence the difference in energy intakes determined by the FFQ and WD. We considered characteristics of the mother (age, body mass index and educational level) and the infant (weight, gender, age solid foods were introduced, existence of non-typical days of diary recording, the number of days between FFQ and WD recording). No predictors of energy difference were found.

\section{Discussion}

A comparison of intakes from the FFQ and WD showed reasonable agreement. This indicates that the intervieweradministered FFQ is a useful tool for estimating energy 
and nutrient intakes in infants about 6 months old. While there were differences in intakes between methods, and the agreement tended to be less good for breast-fed than formula-fed infants, the FFQ provided an estimate of intakes that would be useful in surveys where it is not feasible or appropriate to employ weighed diaries. Specifically, the Spearman rank correlation coefficients, which compared the FFQ's ability to rank an individual's intake of energy and nutrients with that of the WD, indicated good agreement between FFQ and WD values. Adjusting nutrient intakes for energy intake, a measure of nutrient density, strengthened the majority of coefficients $\left(r_{\mathrm{a}}\right)$, demonstrating an increase in agreement between methods. This improvement may arise because energy adjustment may partially correct for measurement error $^{(22)}$.

\section{Comparison with other studies}

Our correlation coefficients compared favourably with other FFQ validation studies for young children. Reported correlation coefficients for energy and nutrients were $0 \cdot 18-0.72$ at 1 year, and $0.26-0.63$ for 1 - to 5-yearolds $^{(23,24)}$; and in a study of 6-month-old infants, comparing nutrient intakes from beverages ${ }^{(25)}$, published coefficients for calcium and vitamin D intakes of 0.49 and 0.80 were similar to ours of 0.62 and 0.86 , respectively.

A comparison of median values of energy intake per kilogram of body weight from the FFQ $(424 \mathrm{~kJ})$ and WD $(370 \mathrm{~kJ})$ with those from the dietary reference values (DRV) for formula-fed infants in the UK $(400 \mathrm{~kJ})^{(26)}$ and energy requirements per kilogram for all infants determined from DLW studies $(339 \mathrm{~kJ})^{(27)}$ showed that the FFQ energy intake was $6 \%$ and $25 \%$ higher than the DRV and DLW values, respectively, and the WD energy intake was 7.5\% lower and $9.1 \%$ higher than the DRV and DLW values, respectively. Thus, both the FFQ and the WD produced median energy intakes per kilogram that are comparable with standard values.

\section{Strengths and weaknesses}

The ability of the FFQ to rank intakes of energy and all nutrients accurately is enhanced by the quality and detail of the information collected and by its short period of recall (1 week), which allows capture of the diet during a period of dietary flux. This FFQ records both the amount consumed and the frequency of consumption of all foods and drinks, and information about brands and types of baby foods and milks used. In addition, trained personnel, able to code baby foods and milks, and demonstrate the 'amount consumed' aids, administered the questionnaire.

Another strength of this study is that the sub-sample comprised a stratified random sample of infants and their families who were representative of the SWS population, which, in turn, is comparable to the UK population ${ }^{(11)}$. However, there was a higher rate of breast-feeding at 6 months (28\%) in our sub-sample and among SWS infants generally $(29 \%)$ than in the UK $(21 \%)^{(28)}$. In addition, possible discrepancies between methods, which cannot be quantified, arise from inbuilt differences between the FFQ and WD: in methodology (retrospective vs. prospective), in time scale ( 7 vs. $4 \mathrm{~d}$ ), and from the WD commencing after the FFQ had been completed.

As in other UK infant dietary studies ${ }^{(6,29)}$, we used an algorithm to estimate human milk intake, which relates length of suckling to intake, for the FFQ and WD. Although regression analysis showed that human milk consumption did not have any consistent effect on difference in energy intakes, the wider distribution of points associated with intakes of breast-fed infants in the Bland-Altman plots for energy and calcium - compared with those who were not breast-fed at 6 months - indicates that the intakes of breast-fed infants were less precisely characterised than those of formula-fed infants.

In common with other studies ${ }^{(23,30)}$, our FFQ tended to produce higher values for actual intakes of energy and nutrients when compared with the WD. The only nutrient with a higher median intake from the WD than from the FFQ was sodium. This results from the gradual change in infant diet over time; compared with the previously collected FFQ, during WD completion more babies were consuming small amounts of non-baby foods, such as cheese and cow's milk, which have higher sodium contents than human milk, formula milks and baby foods. Overall, agreement between actual intakes from the two methods was reasonable, the exception being for vitamin $\mathrm{B}_{12}$; again, the small changes in infant diet, especially the variation in breast-feeding patterns over time, reduced the agreement between methods.

\section{Conclusion}

The comparison of results from the FFQ with those obtained from the WD confirms that our FFQ is a useful tool for assessing the dietary intakes of large groups of infants about 6 months old. While it tended to produce higher median intakes for energy and most nutrients, the magnitudes of the differences were small and the ranking of the infants in terms of intakes was comparable. This interviewer-administered FFQ enables the assessment of energy and nutrient intakes in 6-month-old infants.

\section{Acknowledgements}

We are grateful to the SWS families who have given time and information to the survey. We thank the SWS nurses for data collection, Ken Cox, Patsy Coakley and Vanessa Cox for managing the data, and the SWS Study Group for 
their support. The SWS has received support from the UK Medical Research Council, University of Southampton, Dunhill Medical Trust and British Heart Foundation.

S.E.B. devised the FFQ; H.M.I., K.M.G., C.M.L., S.M.R. and L.D.M. planned the diary study; L.D.M. carried out the fieldwork and, with S.M.R., was responsible for the nutritional analysis; J.P. and H.M.I. performed the statistical analysis. L.D.M. wrote the first draft of the manuscript with contributions from all individual authors.

None of the authors has any conflicting interests.

\section{References}

1. Owen CG, Martin RM, Whincup PH, Davey Smith G \& Cook DG (2005) Effect of infant feeding on the risk of obesity across the life course: a quantitative review of published evidence. Pediatrics 115, 1367-1377.

2. Lanigan JA, Wells JCK, Lawson MS \& Lucas A (2001) Validation of food diary method for assessment of dietary energy and macronutrient intake in infants and children aged 6-24 months. Eur J Clin Nutr 55, 124-129.

3. Alexy U, Kersting M, Sichert-Hellert W, Manz F \& Schöch G (1998) Energy intake and growth of 3- to 36-month-old German infants and children. Ann Nutr Metab 42, 64-74.

4. Michaelson KF (1997) Nutrition and growth during infancy: The Copenhagen Cohort Study. Acta Paediatr 86, Suppl. $420,1-36$.

5. Heinig MJ, Nommsen LA, Peerson JM, Lonnerdal B \& Dewey KG (1993) Intake and growth of breast-fed and formula-fed infants in relation to the timing of complementary foods: the DARLING study. Acta Paediatr 82, 999-1006.

6. Mills A \& Tyler H (1992) Food and Nutrient Intakes of British Infants aged 6-12 months, Appendix V, p. 117. London: HMSO.

7. Stuff JE \& Nichols BL (1989) Nutrient intake and growth performance of older infants fed human milk. $J$ Pediatr 115, 959-968.

8. Dewey KG \& Lonnerdal B (1983) Milk and nutrient intake of breast-fed infants from 1 to 6 months: relation to growth and fatness. J Pediatr Gastroenterol Nutr 2, 497-506.

9. Lande B, Andersen LF, Baerug A, Trygg KU, Lund-Larsen K, Veierod MB \& Bjorneboe G-EAa (2003) Infant feeding practices and associated factors in the first six months of life: the Norwegian infant nutrition survey. Acta Paediatr 92, 152-161.

10. Vähätalo L, Bärlund S, Hannila ML et al., the TRIGR Study Group (2006) Relative validity of a dietary interview for assessing infant diet and compliance in a dietary intervention trial. Matern Child Nutr 2, 181-187.

11. Inskip HM, Godfrey KM, Robinson SM, Law CM, Barker DJP, Cooper C \& the SWS Study Group (2006) Cohort profile: The Southampton Women's Survey. Int J Epidemiol 35, 42-48.

12. Robinson SM, Marriott LD, Poole J, Crozier SR, Borland SE, Lawrence WT, Law CM, Inskip HM, Cooper C \& the SWS Study Group (2007) Dietary patterns in infancy: the importance of maternal and family influences on feeding practice. Br J Nutr 98, 1029-1037.

13. Marriott LD, Foote KD, Bishop JA, Kimber AC \& Morgan JB (2003) Weaning preterm infants: a randomised controlled trial. Arch Dis Child 88, F302-F307.

14. Boggio V, Grossiord A, Guyon S, Fuchs F \& Fantino M (1999) Food consumption of infants and young children in France in 1997. Arch Paediatr 6, 740-747.

15. Harbottle L \& Duggan MB (1993) Dietary assessment in Asian children - a comparison of the weighed inventory and diet history methods. Eur J Clin Nutr 47, 666-672.

16. Black AE, Cole TJ, Wiles SJ \& White F (1983) Daily variation in food intake of infants from 2 to 18 months. Hum Nutr Appl Nutr 37A, 448-458.

17. Paul AA, Black AE, Evans J, Cole TJ \& Whitehead RG (1988) Breastmilk intake and growth in infants from two to ten months. J Hum Nutr Diet 1, 437-450.

18. Holland B, Welch AA, Unwin ID, Buss DH, Paul AA \& Southgate DAT (1995) McCance \& Widdowson's The Composition of Foods. Cambridge: The Royal Society of Chemistry.

19. Willett W (1998) Nutritional Epidemiology, pp. 288-290. Oxford: Oxford University Press.

20. Bland JM \& Altman DG (1986) Statistical methods for assessing agreement between two methods of clinical measurement. Lancet 1, 307-310.

21. Cole TJ (2000) Sympercents: symmetric percentage differences on the $100 \log _{\mathrm{e}}$ scale simplify the presentation of log transformed data. Stat Med 19, 3109-3135.

22. Willett W (2002) Commentary: Dietary diaries versus food frequency questionnaires - a case of undigestible data. Int $J$ Epidemiol 30, 317-319.

23. Andersen LF, Lande B, Arsky GH \& Trygg K (2003) Validation of a semi-quantitative food-frequency questionnaire used among 12-month-old Norwegian infants. Eur J Clin Nutr 57, 881-888.

24. Blum RE, Wei EK, Rockett HRH, Langeliers BS, Leppert J, Gardner JD \& Colditz GA (1999) Validation of a food frequency questionnaire in Native American and Caucasian children 1 to 5 years of age. Matern Child Health J 3, 167-172.

25. Marshall TA, Eichenburger Gilmore JM, Broffitt B, Levy SM \& Stumbo PJ (2003) Relative validation of a beverage frequency questionnaire in children ages 6 months through 5 years using 3-day food and beverage diaries. J Am Diet Assoc 103, 714-720.

26. Department of Health, Committee on Medical Aspects of Food Policy (1991) Dietary Reference Values for Food Energy and Nutrients for the United Kingdom. Report on Health and Social Subjects no. 41, p. 20. London: HMSO.

27. Butte NF (2005) Energy requirements of infants. Public Health Nutr 8, 953-967.

28. Hamlyn B, Brooker S, Lleinikova K \& Wands S (2002) Infant Feeding 2000. London: The Stationery Office.

29. Ong KK, Emmett PM, Noble S, Ness A, Dunger DB \& the ALSPAC Study Team (2006) Dietary energy intake at the age of 4 months predicts postnatal weight gain and childhood body mass index. Pediatrics 117, 503-509.

30. Serdula MK, Alexander MP, Scanlon KS \& Bowman BA (2001) What are preschool children eating? A review of dietary assessment. Annu Rev Nutr 21, 475-498. 